Annuaire suisse de politique de développement

$21 \mid 2002$

Agriculture suisse et mondialisation

\title{
Comment la Suisse appuie la recherche internationale dans le secteur agricole
}

Christina Grieder et Barbara Becker

\section{(2) OpenEdition}

1 Journals

Édition électronique

URL : http://journals.openedition.org/aspd/925

DOI : 10.4000/aspd.925

ISSN : 1663-9669

Éditeur

Institut de hautes études internationales et du développement

Édition imprimée

Date de publication : 1 avril 2002

Pagination : 49-64

ISSN : 1660-5934

\section{Référence électronique}

Christina Grieder et Barbara Becker, « Comment la Suisse appuie la recherche internationale dans le secteur agricole », Annuaire suisse de politique de développement [En ligne], 21 | 2002, mis en ligne le 06 septembre 2012, consulté le 07 septembre 2020. URL : http://journals.openedition.org/aspd/925 ;

DOI : https://doi.org/10.4000/aspd.925 


\title{
COMMENT LA SUISSE APPUIE LA RECHERCHE INTERNATIONALE DANS LE SECTEUR AGRICOLE
}

\author{
Christina Grieder* et Barbara BeCKer ${ }^{* *}$
}

\section{FONCTION}

\section{DE LA RECHERCHE AGRONOMIQUE DANS LA COOPÉRATION AU DÉVELOPPEMENT}

Dans son rapport La situation mondiale de l'alimentation et de l'agriculture (FAO 2000), la FAO prévoit que la population mondiale se stabilisera à 12 milliards d'individus au cours de la seconde moitié du $\mathrm{XXI}^{\mathrm{e}}$ siècle; elle aura ainsi doublé par rapport à l'année 2000. Si l'on veut éradiquer la faim et la malnutrition d'ici là, il faudra tripler la production de denrées alimentaires. Mais la FAO constate que la productivité agricole ralentit depuis 1997; la production agricole des pays en développement a enregistré en 1999 le plus faible taux de croissance depuis 1972. A court terme, les problèmes que pose la sécurité de l'approvisionnement ne seraient pas tellement d'ordre technique, écologique ou démographique; aux yeux de la FAO, ils sont le résultat de formes et de moyens de production inadaptés chez les familles paysannes les plus pauvres, qui ne parviennent même pas à couvrir leurs propres besoins alimentaires. Un autre point important serait le pouvoir d'achat insuffisant des pauvres, citadins aussi bien que ruraux. Le dénuement des populations qui ne travaillent pas dans l'agriculture serait ainsi une conséquence directe de la pauvreté des campagnes et de l'exode rural. La FAO considère cependant qu'une nouvelle révolution verte sera indispensable à plus long terme pour soulager les paysans les plus démunis de ressources, cela en encourageant plus particulièrement des espèces végétales jusqu'ici négligées par l'agriculture (double révolution verte).

La FAO constate que les investissements dans la recherche agricole appliquée, dans la vulgarisation agricole et dans les écoles rurales ont été et restent vitaux pour le développement rural de toutes les économies nationales du monde. Ces observations sont confirmées par des études récentes de l'IFPRI ${ }^{1}$, selon lesquelles des investissements de base - irrigation, recherche agricole, routes de campagne, eau potable et écoles - contribuent plus que tout autre moyen à combattre la faim et la sous-alimentation. Selon les calculs de l'IFPRI, la proportion d'enfants sous-alimentés pourrait avoir diminué en 2020 de $44 \%$ par rapport à 1997 dans les pays en développement qui consacrent 4,9\% de leur budget annuel à ces cinq secteurs. Cela représente un investissement global estimé à quelque 35 milliards de dollars par année (Rosegrant et al. 2001).

Des réformes institutionnelles non accompagnées d'investissements dans ces biens publics ne suscitent aucune croissance économique dans le secteur agricole.

* Spécialiste en agriculture et en recherche agronomique, DDC, Berne.

** Directrice du ZIL (Centre suisse pour l'agriculture internationale), Zurich.

1. L'International Food Policy Research Institute, dont le siège est à Washington, un des centres du GCRAI (Groupe consultatif pour la recherche agricole internationale), <www.ifpri.org $>$. 
La figure 1 visualise les interactions produites par ces types d'investissements: elle montre qu'il est important d'investir dans la formation et la vulgarisation pour combattre la pauvreté et assurer la sécurité alimentaire, mais aussi que les investissements des pouvoirs publics dans la recherche agricole donnent alors des résultats probants. Indirectement, ils stimulent aussi d'autres domaines de recherches et celles qui sont menées par d'autres partenaires (figures 2 et 3). La tendance actuelle est pourtant inverse: l'aide publique au développement a régulièrement diminué dans le secteur agricole au cours des années 1990 jusqu'à aujourd'hui.

\section{Figure 1: Amélioration de la productivité en fonction du développement technologique}

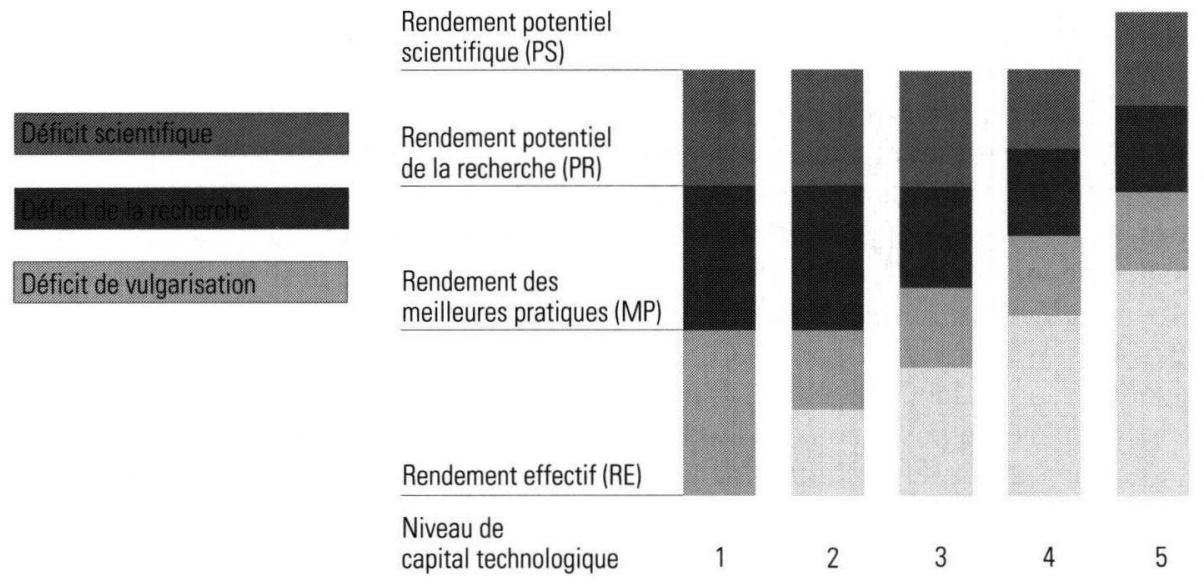

Source: FAO, La situation mondiale de l'alimentation et de l'agriculture 2000, p. 251.

Note: MP-RE, le déficit de vulgarisation, est la différence entre le rendement moyen effectif et les rendements qui pourraient être obtenus avec des pratiques exemplaires. Les programmes de vulgarisation et les investissements dans l'infrastructure sont destinés à combler ce déficit.

PR-MP, le déficit de recherche d'adaptation, est la différence entre le rendement obtenu au moyen des meilleures pratiques connues et celui qui pourrait être obtenu avec des pratiques améliorées grâce à la recherche d'adaptation. Les programmes de recherche appliquée, s'ils réussissent, combleront ce déficit.

PS-PR, le déficit scientifique, est la différence entre le rendement correspondant aux pratiques exemplaires améliorées et le rendement obtenu si la recherche appliquée était appuyée par la recherche fondamentale. Pour combler ce déficit, il faut que la recherche fondamentale vienne soutenir la recherche d'adaptation.

On trouve au niveau CT1 les pays qui n'ont encore rien entrepris dans les domaines de la recherche scientifique et de la vulgarisation; et les recherches qui produisent des technologies adaptées à d'autres régions ne contribuent pas à résoudre les problèmes des pays ou régions situés à ce niveau. La plupart des progrès se réalisent dans les régions qui ont déjà atteint le stade CT2, auquel se trouvaient les pays de l'Asie méridionale à l'époque de la révolution verte; ils ont ainsi profité de ces impulsions scientifiques et atteint le niveau CT3. Actuellement, plusieurs pays d'Asie et d'Amérique latine en sont presque au niveau CT4.

Dans son Rural Poverty Report 2000, l'IFAD $^{2}$ considère également qu'une amélioration durable de la productivité agricole chez les petits paysans constitue le 
meilleur moyen de combattre la pauvreté. Dans les pays les plus démunis (too many people are too poor), cela devrait se combiner à un processus de redistribution ([empowerment] through higher shares, access and control of appropriate assets, institutions, technologies and markets). Plus de la moitié des indigents de la planète dépendent de l'agriculture et du travail dans ce secteur, donc d'un développement dynamique de celui-ci. Pourtant, l'indispensable appui financier des pouvoirs publics a régressé entre 1987 et 1998 de plus de deux tiers dans ce domaine impliquant toute une série d'investissements: écoles, infrastructure rurale, santé, développement technologique, recherche, développement institutionnel et accès aux marchés. C'est pourquoi l'IFAD demande, dans son rapport, que l'on réinjecte davantage de ressources dans le développement rural pour combattre efficacement la pauvreté.

Figure 2: Le rôle de la recherche publique nationale et internationale ${ }^{*}$

Relations entre secteurs public et privé à divers niveaux géographiques pour la production de biens basés sur l'innovation scientifique

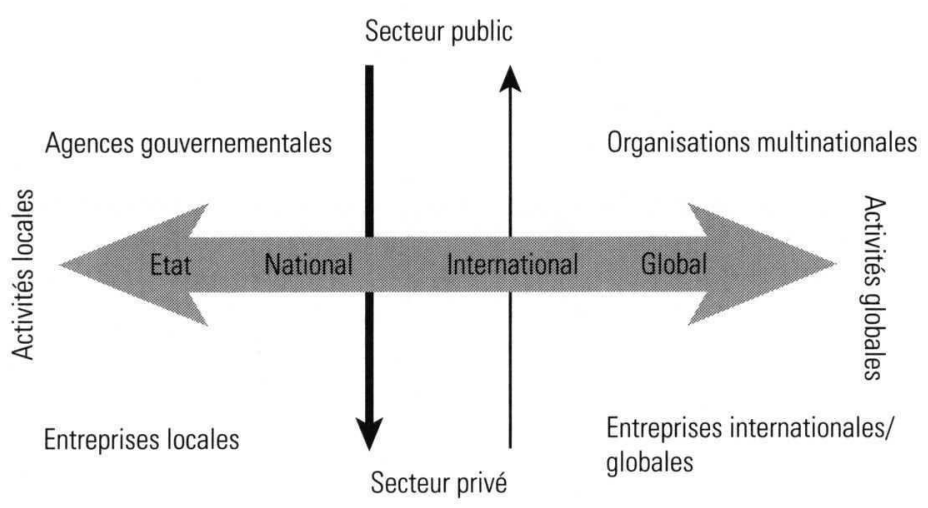

Source: Dalrymple (2001)

Note: on admet que chaque quadrant interagit avec tous les autres, bien que le secteur privé obtienne traditionnellement davantage du secteur public que le contraire; mais cette situation est en train de changer dans la mesure où le secteur privé gagne en importance dans certains secteurs de la recherche scientifique et détient de plus en plus de droits de propriété intellectuelle.

* Les centres de recherche agricole internationaux sont des acteurs globaux à placer dans le quadrant supérieur droit.

Dans son Rapport sur le développement dans le monde 2000-2001. Combattre la pauvreté, la Banque mondiale donne à entendre que les investissements du secteur public contre les maladies carentielles et infectieuses et pour l'accroissement de la productivité agricole constituent les principaux biens publics dont peuvent bénéficier les plus démunis. Elle considère ainsi que c'est dans ces domaines que l'on peut le mieux combattre la pauvreté. L'économie privée ne produit pratiquement pas de biens publics nationaux ou internationaux pour l'agriculture ou la santé, par manque de stimulants dans ce sens. Une lutte efficace contre la pauvreté requiert donc - outre la coopération entre Etats - beaucoup d'investissements publics. La Banque mondiale préconise par conséquent un regain d'investissements dans les secteurs de l'agriculture et de la santé.

Divers scientifiques se sont également exprimés ces dernières années sur le rôle de la recherche et de la production agricoles dans la lutte contre la pauvreté. Selon des économistes tels que Jeffrey Sachs, de l'Université d'Harvard, ou 
Michael Lipton, de l'Université du Sussex, les travaux des centres de recherche agronomique du GCRAI (Future Harvest Centers) produisent des biens publics internationaux de très grande valeur pour les populations dans le besoin. Ils estiment particulièrement important de développer et d'exploiter de nouvelles technologies visant à trouver des solutions efficaces aux problèmes des plus démunis et à accroître la sécurité alimentaire. Tous deux critiquent le fait que les investissements de la coopération internationale dans la recherche agricole et l'espace rural aient diminué, alors que leur rentabilité est très élevée.

Figure 3: Relations entre recherche publique et privée, fondamentale et appliquée

Organisation de la recherche scientifique et du développement technologique dans les secteurs public et privé (modèle visualisé par un système de coordonnées rectangulaires)

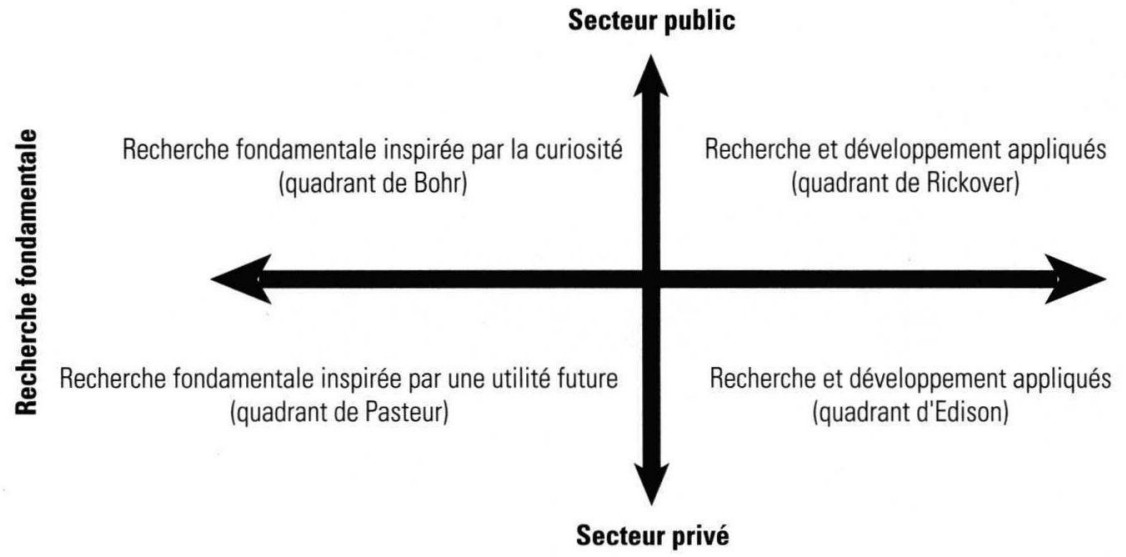

Source: Dalrymple (2001), adapté par Vernon W. Ruttan, Technology, Growth and Development: An Induced Innovation Perspective, Oxford University Press, 2001, p. 237 (réadapté par Ruttan à partir d'autres sources).

Il faut compter au moins une bonne dizaine d'années, voire jusqu'à vingt ans, entre le moment où débutent des travaux de recherche et celui où leurs résultats sont effectivement appliqués par les paysans. L'approvisionnement alimentaire de la génération à venir dépend donc en partie des investissements actuels dans la recherche. Il existe aujourd'hui, sur le papier, un équilibre global entre offre et demande de denrées alimentaires, dont nous n'avons pas lieu de tirer vanité; il sera indispensable d'investir pour l'avenir. Selon la FAO, plus de 800 millions de personnes - davantage que les habitants d'Amérique du Nord et d'Europe occidentale réunis - souffrent aujourd'hui encore de malnutrition et de sousalimentation. Cela bien que la population actuelle soit deux fois et demie plus nombreuse que dans les années 1960 , et que le nombre des affamés ait diminué au cours de la même période aussi bien proportionnellement qu'en chiffres absolus $^{3}$.

Ce résultat remarquable est dû en grande partie à la recherche agronomique. Actuellement, les familles paysannes des pays en développement, à côté de

3. FAO, La situation mondiale de l'alimentation et de l'agriculture 2000 , p. 305 . 
leurs variétés traditionnelles, cultivent plus de 300 variétés de blé et de riz, et plus de 200 variétés de maïs sélectionnées par les centres du GCRAI ${ }^{4}$. La productivité du blé et du riz a doublé. La valeur du blé produit dans des pays en développement a augmenté de plus de 1,8 milliard de dollars au cours des trente dernières années; la production de riz en Inde a presque triplé sur la même superficie $^{5}$. Cette amélioration des rendements a également fait baisser les prix au consommateur des aliments de base, qui sont ainsi devenus plus abordables pour les pauvres. Cela signifie donc que les efforts entrepris dans le cadre de la «révolution verte» des années 1960 et 1970 ont été très fructueux, avec des gains de productivité qui ont largement compensé la progression rapide de la population mondiale.

La pression exercée par la demande a également entraîné une forte progression de la production animale, rendue possible par la recherche et l'évolution technologique (surtout dans les domaines de la sélection, de l'alimentation animale et du contrôle des maladies, Fuglie et al. 2000). Dans les pays en développement, cette production a augmenté au cours des vingt dernières années dans la proportion de $5 \%$ en moyenne pour la viande, et de $3 \%$ pour le lait. La valeur marchande de cet accroissement dépasse la plus-value de production céréalière obtenue par la «révolution verte» (Delgado et al. 2001).

Mais aujourd'hui on jette aussi un regard critique sur les réussites de la « révolution verte»: cette productivité accrue - obtenue surtout par des variétés à haut rendement en culture intensive avec des engrais et des produits phytosanitaires, ainsi qu'en intensifiant la production animale - a provoqué dans les systèmes agro-écologiques des dégâts que l'on ne peut plus tolérer de nos jours. Il sera toujours nécessaire d'améliorer le rendement agricole, mais en respectant l'environnement de manière à produire durablement de quoi nourrir une population mondiale qui continue d'augmenter. Il faudra en même temps que les résultats de ces travaux de recherche et de développement profitent avant tout aux populations rurales des pays les plus pauvres, pour qu'elles sortent enfin de la misère. Les efforts entrepris depuis une quinzaine d'années pour promouvoir une production agricole durable ont porté leurs fruits: c'est ainsi que le volume de pesticides utilisés dans les pays en développement a beaucoup diminué grâce aux méthodes de la protection intégrée des cultures. Ces méthodes ont été mises au point notamment par divers organismes - centres du GCRAI, ICIPE ${ }^{6}$, $\mathrm{CABI}^{7}$, FAO et instituts de recherche du Nord - en collaboration avec des partenaires de pays en développement. Le contrôle des insectes qui ravageaient les cultures de manioc en Afrique apporte aujourd'hui une plus-value de quelque 400 millions de dollars par année (cf. note 5). Depuis les années 1980 on se préoccupe également davantage de préserver la diversité des plantes utilisées par l'agriculture afin d'exploiter les ressources génétiques locales tout en obtenant

4. Les centres internationaux de recherche agronomique du GCRAI (Future Harvest Centers), ainsi que les centres associés tels que l'ICIPE, appartiennent au quadrant supérieur droit de la figure 3 . Il incombe à la recherche financée par les collectivités publiques d'aborder des sujets et des problèmes qui n'intéressent pas la recherche financée par l'économie privée - par exemple la production agricole dans des zones marginales et pour les paysans économiquement faibles des pays en développement.

5. <www.cgiar.org/who/wwa_impact.html>.

6. International Centre of Insect Physiology and Ecology, Nairobi, Kenya, <www.icipe.org $>$.

7. Centre for Agriculture and Bioscience International, Wallingford, Royaume-Uni, <www.cabi.org $>$. 
de meilleurs rendements (résistance à la sécheresse et au froid, teneur en protéines, etc., cf. note 5). Il s'agit notamment d'obtenir régulièrement de bonnes récoltes en réduisant les apports d'engrais (low input agriculture).

Outre les considérations d'ordre écologique, la recherche agronomique internationale s'intéresse toujours davantage au contexte économique et socioculturel de l'évolution technologique. L'IFPRI, par exemple, s'est basé sur une modélisation économique pour proposer un programme, Vision 2020, qui indique des tendances et des scénarios sur la manière dont demande et production vont évoluer dans le secteur agricole au cours des deux décennies à venir. Ces études montrent entre autres comment l'urbanisation galopante des pays en développement accroît la demande de produits animaux, et quelles mesures il faudra prendre pour satisfaire cette demande. L'IFPRI estime que les petites exploitations paysannes profiteront de ces marchés en expansion si trois conditions sont remplies, à savoir:

- réformes des marchés avec investissements en faveur des petits paysans tout en évitant de subventionner les grosses exploitations;

- institutions aidant les paysans à livrer en temps utile et à se conformer aux normes internationales de qualité et de sécurité;

ऽ mise à disposition de biens publics tels que recherche, vulgarisation et infrastructure ${ }^{8}$.

Cet élargissement de l'approche technologique s'est accompagné de la prise de conscience qu'il est essentiel d'associer les divers acteurs concernés. On a élaboré des sujets et des méthodes de recherche incluant la participation des familles paysannes ainsi que des expériences menées sur les exploitations agricoles. Au niveau stratégique, il est maintenant admis que la recherche agronomique internationale doit résulter d'efforts impliquant tous les milieux concernés. Cette constatation a débouché sur le regroupement de toutes les parties prenantes au sein d'un Global Forum for Agricultural Research (GFAR) ${ }^{9}$, qui anime le dialogue et la collaboration entre centres du GCRAI, économie privée, associations paysannes, universités et institutions de recherche nationales, et organisations de la société civile. Tous ces milieux peuvent contribuer d'une manière ou d'une autre au combat contre la pauvreté et la faim (figures 2 et 3 ).

Les progrès réalisés par la biologie moléculaire ont notamment pour effet de rapprocher dans le domaine génétique d'une part la recherche privée des pays industrialisés et de l'autre les centres du GCRAI. Il en résulte des synergies et des possibilités de collaboration inédites. Le bien public global qu'élaborent par exemple les centres du GCRAI sous forme de variétés végétales améliorées sont également à la disposition d'utilisateurs de l'hémisphère Nord: l'Amérique du Nord, notamment, a déjà tiré parti de variétés améliorées de blé et de riz. Et les pays d'autres bailleurs de fonds profitent également des activités de recherche et de développement agronomiques. De ce fait, les pays qui investissent dans les centres du GCRAI apportent un soutien indirect au développement de leur propre agriculture (cf. figure 2). La DDC considère que les programmes de

8. <www.ifpri.org/2020/welcome.htm>.

9. Le GFAR (<www.gfar.org $>$ ) tient un secrétariat auprès de la FAO pour coordonner ses activités dans le monde, structurées en six forums régionaux. 
recherche du GCRAI ont une importance stratégique en raison de leur fonction de lien entre la recherche scientifique du Nord et les programmes des pays en développement, et aussi du fait qu'ils stimulent la collaboration Sud-Sud.

A la suite des critiques qu'elles ont émises à l'égard de la «révolution verte», les organisations non gouvernementales $(\mathrm{ONG})$ sont devenues des partenaires de la recherche agricole internationale dont le point de vue est de plus en plus respecté. Ces représentants de la société civile s'emploient à mettre en valeur le savoir local et les techniques traditionnelles, les méthodes de l'agriculture écologique et intégrée, l'importance sociale des petites exploitations agricoles. Le fait de collaborer avec des ONG favorise l'intégration des connaissances locales et globales, la mise en relation des divers niveaux de recherche stratégique et appliquée (figures 2 et 3 ).

Depuis quelques années, la recherche agricole internationale s'intéresse plus particulièrement à l'évolution de l'Afrique subsaharienne: $42 \%$ des investissements effectués en 2000 par les centres du GCRAI sont allés à cette région. Le GCRAI a consacré au cours de ses vingt premières années environ $54 \%$ de ses dépenses de recherche à l'amélioration de la productivité agricole. Cette proportion est ramenée à $36 \%$ depuis le début des années 1990, et l'on investit d'autant plus dans les secteurs environnement, biodiversité et recherche politique. Depuis 1972, les centres du GCRAI destinent par ailleurs $21 \%$ de leurs investissements aux instituts nationaux de recherches agronomiques (NARI) ${ }^{10}$ des pays en développement.

Les NARI des différents pays ont évolué de façon très variable ces dernières années. Alors que certains pays émergents (p. ex. Brésil, Chine, Inde) ont d'importantes institutions de recherche et du personnel qualifié qui leur permettent de travailler dans plusieurs disciplines, d'autres - surtout ceux de l'Afrique subsaharienne - sont confrontés à de gros problèmes par manque de ressources financières, humaines et matérielles. Il en résulte des partenariats de recherche adaptés aux particularités de ces acteurs.

\section{LA CONTRIBUTION DE LA SUISSE À LA RECHERCHE AGRONOMIQUE INTERNATIONALE}

La contribution de la Suisse à la recherche internationale dans le secteur agricole constitue depuis de nombreuses années un volet important de la coopération suisse au développement. Elle s'appuie pour ce faire sur toute une série de partenaires et d'institutions dont la vocation est d'encourager la recherche. Il en résulte également une grande diversité dans les objectifs, les contenus, les groupes cibles et les formes de coopération (cf. figure 4). En Suisse même, des organismes d'encouragement aussi bien que des institutions de recherche participent à cette tâche globale, contribuant par divers moyens - apports financiers, conseil et dialogue politiques, développement technologique - aux objectifs communs de combattre la pauvreté, d'assurer la sécurité alimentaire et de préserver l'environnement. Le protagoniste de ces activités en Suisse est la Direction du développement et de la coopération (DDC). 
Figure 4: Acteurs et cibles de la recherche agronomique internationale en Suisse

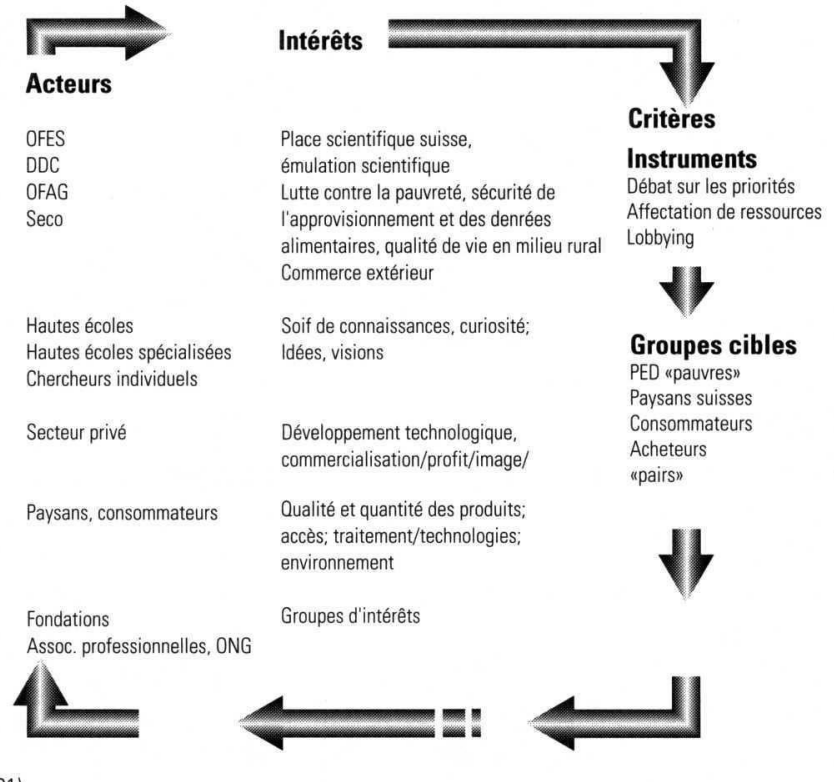

Source: Becker (2001)

\section{LES INVESTISSEMENTS DE LA DDC DANS LA RECHERCHE AGRICOLE INTERNATIONALE}

Pour accomplir son travail, la DDC se réfère d'une part à la Loi fédérale sur la coopération au développement et l'aide humanitaire internationales (1976), de l'autre aux Lignes directrices Nord-Sud adoptées par le Conseil fédéral en 1994. Le premier document décrit le mandat de la DDC, qui est entre autres de combattre la pauvreté - plus particulièrement dans les zones rurales des pays en développement; le second souligne l'importance de ménager les ressources naturelles. Dans sa Stratégie 2010 achevée au cours de l'été 2000, la DDC réaffirme que l'utilisation durable des ressources naturelles restera une de ses priorités. Les engagements pris par la Suisse en matière de coopération internationale - lors du Sommet mondial de l'alimentation en 1996 et dans le cadre des conventions relatives à la diversité biologique, aux changements climatiques et à la lutte contre la désertification - sont respectés et mis en œuvre de manière systématique par la DDC. Celle-ci se réfère donc à cet ensemble de normes et d'engagements pour ses contributions à la recherche agronomique internationale.

La DDC considère qu'il est important de soutenir la recherche agricole à l'intention des pays en développement pour atteindre les objectifs de sa nouvelle stratégie d'utilisation durable des ressources naturelles. Par ailleurs, la production agricole constitue à ses yeux un moteur déterminant pour l'amélioration des revenus et de l'emploi. Sa Politique sectorielle dans le domaine agricole (1999) expose comment la direction prise par le développement agricole dépend du contexte économique et culturel, des ressources (naturelles et autres), de l'accès qu'y ont les familles paysannes et des conditions données par la politique (agricole). Elle souligne également que les trois objectifs de la DDC en matière de développement - diminuer la pauvreté, assurer la sécurité alimentaire, pro- 
mouvoir une utilisation durable des ressources naturelles - ne peuvent être poursuivis utilement que de manière cohérente et coordonnée.

La DDC investit depuis le début des années 1970 dans la recherche agronomique pour les pays en développement, avec au départ les Future Harvest Centers du GCRAI comme principaux partenaires. Tandis que la FAO est un interlocuteur important pour les questions politiques touchant à la production agricole intégrée, à l'utilisation durable du sol et de l'eau, à la diversité des espèces (préservation, accès, exploitation). Ces dix dernières années, la DDC a intensifié sa collaboration avec le CABI, avec l'ICIPE (actif essentiellement en Afrique), et, de plus en plus, avec des ONG qui s'occupent de recherche. La DDC participe aux centres de recherches par des apports non affectés (budgets d'exploitation, contributions à des programmes) aussi bien qu'affectés (financement de projets). Ce sont en général les unités opérationnelles de la DDC en Afrique, en Amérique latine et en Asie qui s'occupent de financer des projets; ceux-ci portent pour la plupart sur la sélection et l'adaptation de céréales telles que riz et maïs, de tubercules et racines tels que pommes de terre, patates douces et manioc, de haricots et autres légumineuses, à cultiver dans des conditions difficiles - sols pauvres en substances nutritives, sécheresse, humidité, froid. Ces dernières années, la Suisse a soutenu plusieurs projets de recherche concernant des méthodes de culture sur pente et en altitude, et pour le maintien de la diversité biologique. La DDC a investi en 2001 environ 23 millions de francs dans la recherche agronomique du GCRAI, dont une petite moitié non affectée.

Un critère important, pour ces investissements, est de créer des biens publics globaux. Sous les auspices de la FAO, les centres du GCRAI tiennent à la disposition de la communauté internationale, dans leurs banques de gènes, plus de 600’000 entrées végétales (espèces, variétés, plantes sauvages apparentées aux espèces utilisées par l'agriculture). Ce potentiel a une valeur inestimable pour la sélection végétale et constitue le noyau d'un Engagement international sur les ressources phytogénétiques pour l'alimentation et l'agriculture. La Convention sur la diversité biologique a confié à la FAO le soin d'élaborer cet accord international. Une conférence technique internationale tenue à Leipzig en 1996 a adopté un plan d'action global ayant pour objet la sauvegarde et l'utilisation durable des ressources phytogénétiques pour l'alimentation et l'agriculture. Les collections ex situ que contiennent les banques de matériel génétique de nombreux pays et les centres du GCRAI constituent un élément essentiel de ce plan d'action, étant entendu qu'il s'agit de mettre ces ressources génétiques plus particulièrement à la disposition des pays en développement. La DDC estime ainsi justifié d'apporter aux centres du GCRAI des contributions non affectées dans le cadre des engagements qu'a pris la Suisse en ratifiant la Convention sur la diversité biologique, à côté de la grande importance que revêtent ces centres pour la sécurité alimentaire.

La DDC s'occupe de biotechnologies dans le cadre de l'Agenda 21 et de la mise en œuvre de la Convention sur la diversité biologique; ses préoccupations prioritaires sont ici les politiques à adopter, l'accès aux technologies, ainsi que des questions d'éthique et d'équité. Elle s'emploie à mettre en valeur les avantages possibles des biotechnologies et à en réduire les risques socio-économiques et écologiques, en agissant sur trois axes: elle renforce les capacités de mettre sur pied des politiques en matière de biotechnologies, soutient l'aptitude des pays 
en développement à exploiter ces technologies et à faire de la recherche en la matière, encourage les partenariats scientifiques et les transferts de technologie.

Les recherches du secteur privé - surtout les résultats de la recherche fondamentale et des biotechnologies - présentent un certain intérêt pour les pays en développement, ce qui conduit la DDC à soutenir les efforts qu'entreprend la recherche agronomique internationale pour diffuser ou exploiter ce savoir. Le fait de collaborer avec des organismes de recherche du secteur privé confère à la question des brevets une importance qu'elle n'avait pas dans le passé. La protection apportée par la propriété intellectuelle, notamment sous forme de brevets, stimule l'économie privée à investir dans la recherche scientifique. Par conséquent, les institutions privées de recherche agronomique ne seront d'accord de coopérer avec les centres du GCRAI et d'autres organismes internationaux que si leur concurrence dans le Nord n'a pas également accès aux connaissances et aux technologies qu'elles auront développées. Elles sont en revanche disposées à offrir leur savoir pour des applications dans les pays en développement. Il apparaît ainsi que les centres internationaux de recherche agricole devront également breveter les résultats de leurs collaborations avec le secteur privé $^{11}$, afin que les pays en développement puissent accéder durablement à des connaissances et des résultats protégés. En tout état de cause, les centres du GCRAI et les autres organismes analogues ne concluent des accords de ce genre que s'ils obtiennent des droits de licence sans restrictions pour leur travail et l'application dans des pays en développement. Les produits de ces partenariats sont alors des biens publics pour les pays en développement, même s'ils n'ont plus un caractère universel.

Le Service international pour l'acquisition d'applications agrobiotechnologiques (ISAAA) ${ }^{12}$ s'occupe de transmettre des technologies et apporte son soutien pour les questions de propriété intellectuelle qui peuvent en résulter (licences); la DDC coopère avec cette organisation depuis 1996. L'ISAAA a mis en place avec cet appui un réseau de diffusion de connaissances qui est particulièrement actif dans le Sud-Est asiatique (Thailande, Vietnam, Philippines, Chine). Cette organisation collabore étroitement avec la recherche agronomique privée et constitue ainsi un trait d'union important entre NARI et milieux économiques.

\section{ACTIVITÉS DE RECHERCHE AGRONOMIQUE INTERNATIONALE EN SUISSE}

La DDC encourage également la recherche agricole suisse à coopérer avec les pays en développement, les centres du GCRAI et d'autres organismes de recherche internationaux. Ce soutien prend différentes formes: certains de ses «programmes par pays» appuient des actions bilatérales telles que le programme Indo-Swiss Collaboration in Biotechnology en Inde; la DDC passe par le Centre suisse pour l'agriculture internationale (ZIL), dont le siège se trouve à l'EPF de Zurich, pour réaliser certains projets de coopération avec des centres de recherche internationaux; enfin, le Swiss Forum for International Agricultural Research (SFIAR) entretient le dialogue entre les divers partenaires en Suisse qui s'intéressent à la recherche agronomique sur le plan international.

11. Les centres du GCRAI ont déposé jusqu’à présent moins de dix demandes de brevets.

12. International Service for the Acquisition of Agri-biotech Applications, <www.isaaa.org >. 
Le Centre suisse pour l'agriculture internationale (ZIL) ${ }^{13}$ regroupe la plupart des instituts et des scientifiques de l'EPFZ qui enseignent et font de la recherche dans le secteur agricole. Comme le ZIL forme une interface entre école polytechnique et recherche agronomique à l'intention des pays en développement, le financement de ses activités est assuré conjointement par l'EPFZ et la DDC. Seule faculté agronomique en Suisse, l'EPFZ exerce une fonction stratégique importante pour la coopération avec les centres de recherche dans ce secteur.

Le ZIL s'est concentré dès sa création sur quelques pôles d'activité. Son programme le plus important est consacré au manioc, avec, depuis 1993, une dizaine de projets de recherche pour un volume global dépassant 2 millions de francs. Leurs thèmes essentiels sont l'étude, au niveau de la biologie moléculaire, des fondements de la résistance aux virus, la lutte biologique contre les parasites, les apports en éléments fertilisants, les cultures mixtes, ainsi que les questions socio-économiques relatives à la consommation et à la commercialisation. Les principaux partenaires de recherches du ZIL sont le CIAT ${ }^{14}$ (un centre du GCRAI) et l'IITA ${ }^{15}$.

Par ailleurs, le ZIL s'intéresse depuis qu'il existe à la production laitière des petites exploitations paysannes et à la commercialisation de ce produit. Concentrées les premières années sur l'Inde - dans le cadre d'une coopération de longue haleine qu'y mène la DDC dans le domaine de la production animale -, ses activités se sont déplacées plus récemment vers l'Afrique orientale. Pour ce type de recherches, il collabore surtout avec l'ILRI ${ }^{16}$ et l'IFPRI ${ }^{17}$.

Ses travaux pour la lutte intégrée contre les parasites (integrated pest management, IPM) sont regroupés depuis 1997 dans un pôle d'activité séparé. Ils portent sur les cultures de manioc et d'igname, ainsi que sur la protection après récolte des légumineuses à grains. Ses principaux partenaires dans ce domaine sont le CIAT et le Centre suisse de recherche scientifique (CSRS) en Côte d'Ivoire.

Les légumineuses destinées à l'amélioration des sols constituent enfin, depuis deux ans, le quatrième pôle d'activité actuel du ZIL, avec des études sur les amendements et sur la fertilisation au niveau de ses mécanismes de biologie moléculaire. Ces travaux se réalisent en collaboration avec l'ICRAF ${ }^{18}$.

Dans le cadre d'un programme de relève géré par le ZIL, la DDC appuie en outre les travaux de scientifiques suisses qui font de la recherche agricole et forestière dans des pays en développement. Ce Research Fellow Partnership Programme (RFPP) offre chaque année à deux ou trois personnes l'occasion de travailler avant ou après leur thèse dans des centres internationaux de recherches agronomiques. Il est ouvert à des candidats et candidates de toutes les hautes écoles et institutions de recherche de la Suisse qui souhaitent réaliser un projet commun avec un de ces centres internationaux. Les 15 projets soutenus jusqu'à présent englobent tous les secteurs des sciences agronomiques et

13. <www.zil.ethz.ch>.

14. Centro Internacional de Agricultura Tropical, Cali, Colombie, <www.ciat.org $>$.

15. International Institute of Tropical Agriculture, Ibadan, Nigeria, <www.iita.org $>$.

16. International Livestock Research Institute, Nairobi, Kenya, <www.ilri.org $\rangle$.

17. International Food Policy Research Institute, Washington, D.C., <www.ifpri.org $>$.

18. International Centre for Research in Agroforestry, Nairobi, Kenya, <www.icraf.org >. 
forestières, depuis l'établissement de priorités dans les recherches en biotechnologies dans certains pays en développement jusqu'à l'exploitation durable des forêts de noyers naturelles au Kirghizistan. Les centres partenaires sont le CIAT, le CIFOR $^{19}$, le CIMMYT ${ }^{20}$, le CIP ${ }^{21}$, l'IITA, l'IRRI ${ }^{22}$ et l'ISNAR ${ }^{23}$ (ZIL 2000).

Par ailleurs, 1'EPFZ poursuit d'importants travaux de recherche en dehors des activités financées par la DDC dans le cadre de son mandat politique. Ces activités s'inscriraient dans les quadrants gauches de la figure 3, sous Recherche fondamentale inspirée par la curiosité et inspirée par une utilité future. Un excellent exemple à cet égard est le Golden Rice dû à l'initiative d'un chercheur motivé et convaincu de l'EPFZ, et devenu depuis lors un programme de recherche et développement d'envergure mondiale (Potrykus 2001 ; IRRI 2001). Ses activités ont maintenant passé de la biologie moléculaire à la garantie des droits de propriété intellectuelle dans le domaine public.

Les travaux qui ont abouti au Golden Rice sont représentatifs de la ligne de conduite adoptée par l'EPFZ: poursuivre des recherches high-tech à caractère très fondamental dans des domaines qui promettent explicitement des applications socialement souhaitables (charte de l'EPFZ 1996). Mais l'Ecole polytechnique de Zurich travaille aussi à des niveaux beaucoup plus proches de la mise en œuvre pratique, par exemple en acceptant des mandats de recherche de l'économie privée en relation avec des pays en développement (tels qu'études de marché, développement de produits) ou des études parallèles à des projets de coopération au développement (p. ex. en économie forestière).

La recherche appliquée (figure 3: Recherche et développement appliqués) est une tradition des hautes écoles spécialisées. En Suisse, les activités internationales de cet ordre dans le domaine agricole sont surtout le fait de la Haute Ecole suisse d'agronomie (HESA) à Zollikofen, qui fait partie de la Haute Ecole spécialisée bernoise. Ses enseignants participent par exemple à des évaluations de projets et à des missions analogues sous mandat de la DDC ou d'autres institutions de la coopération au développement. L'HESA assume aussi, toujours pour le compte de la DDC, le service d'information infoagrar avec la plate-forme Internet Swissnode (<www.sfiar.infoagrar.ch>), service en ligne du Swiss Forum for International Agricultural Research (SFIAR).

Des entreprises privées, des organisations non gouvernementales (ONG) et des institutions de recherche non universitaires constituent d'autres acteurs suisses de la recherche agricole internationale. Ces trois groupes appartiennent à la moitié droite de la figure 3, donc plutôt dans le Développement technologique que dans la Recherche fondamentale. Les principaux acteurs de l'économie sont Syngenta et la fondation du même nom, qui s'occupent tous deux de protection phytosanitaire et de semences. Du côté des ONG, Intercooperation réalise des projets agricoles et sylvicoles dont certains comportent des éléments de recherche appliquée. Parmi les organismes de recherche extra-universitaires, le Forschungsinstitut für biologischen Landbau (FiBL) collabore avec des institu-

19. Centre for International Forestry Research, Bogor, Indonésie, <www.cifor.org $>$.

20. Centro Internacional de Mejoramiento de Maiz y Trigo, Mexico, Mexique, <www.cimmyt.org >

21. Centro Internacional de la Papa, Lima, Pérou, <www.cip.org >.

22. International Rice Research Institute, Los Baños, Philippines, <www.irri.org >

23. International Service for National Agricultural Research, La Haye, Pays-Bas, <www.isnar.org >. 
tions analogues de différents pays en développement (p. ex. avec la Chine en arboriculture biologique).

Cette énumération incomplète devrait donner une idée de la diversité institutionnelle et thématique qui caractérise la coopération entre la Suisse et la recherche agricole internationale (cf. IUED 2000). Tous les organismes intéressés se sont regroupés depuis 1998 au sein du Swiss Forum for International Agricultural Research (SFIAR), lequel assure contacts et dialogue entre ces diverses instances afin de promouvoir coopération, synergies et complémentarités entre les acteurs de la recherche internationale en Suisse dans le domaine agricole, et d'améliorer ainsi l'efficacité des investissements suisses et des développements qui doivent en résulter. Le SFIAR permet à la Suisse de participer au développement des réseaux qui, partout dans le monde, relient de plus en plus les milieux intéressés aux niveaux planétaire aussi bien que régional ou national. La Suisse fait ainsi partie de l'European Forum for Agricultural Research for Development (EFARD) et, à l'échelle mondiale, du Global Forum for Agricultural Research (GFAR).

Il est indispensable que l'ensemble des acteurs de tous les milieux concernés unissent leurs forces face au défi que représentent la faim et la pauvreté pour la recherche agricole internationale. Et il ressort de tous les travaux évoqués ici que la recherche joue un rôle clé à cet égard. L'accomplissement de cette tâche requiert de gros moyens sur tous les plans - humain, technologique, financier -, et la Suisse est bien placée pour y apporter sa contribution. Il lui incombera de poursuivre résolument ses efforts pour relever ce défi. 
Annexe 1: Investissements de la DDC dans la recherche agricole 1990-2000 (en millions de francs)

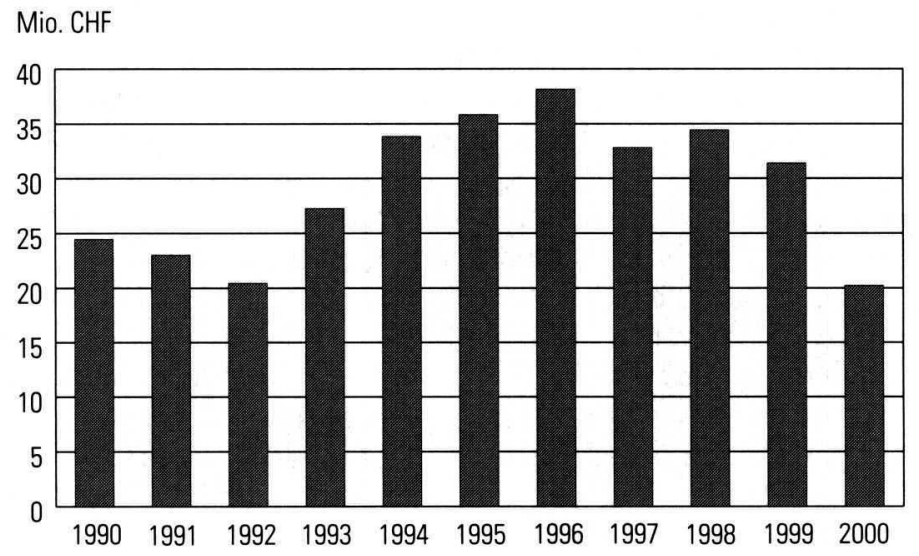

Source: DDC (Catherine Graf/Christina Grieder), 2001. 


\section{Annexe 2: Investissements de la DDC dans la recherche agricole 1990-2000: répartition entre diverses organisations internationales}

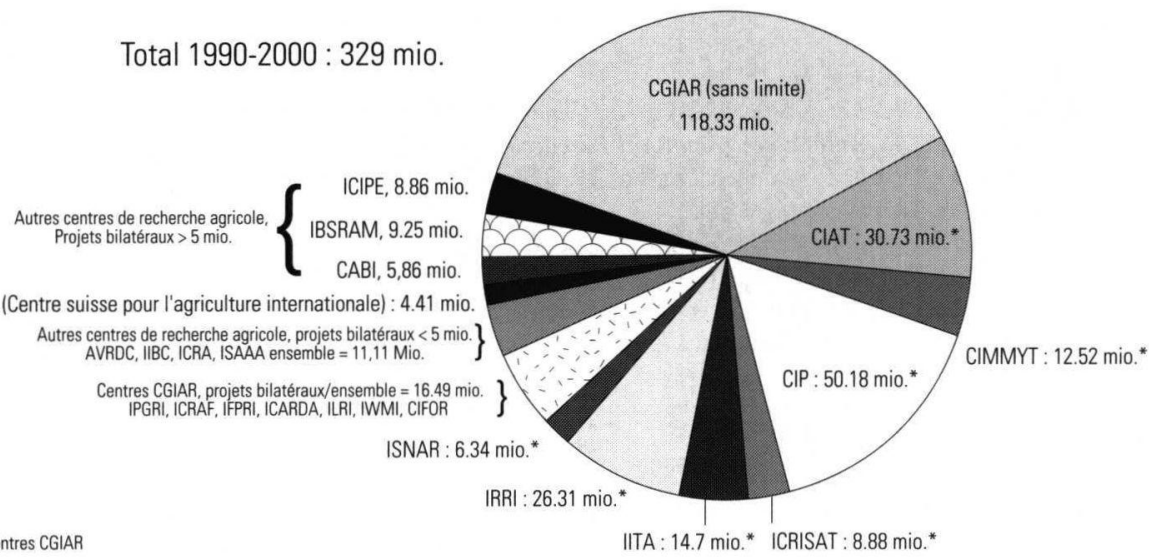

Source: DDC (Catherine Graf/Christina Grieder), 2001.

AVRDC: Asian Vegetable Research and Development Center

$\mathrm{CABI}$ : Centre for Agriculture and Biosciences International

CGIAR: Consultative Group on International Agricultural Research

CIAT: Centro Internacional de Agricultura Tropical

CIFOR: Center for International Forestry Research

CIMMYT: International Maize and Wheat Improvement Center - Centro Internacional de Mejoramiento de Maíz y Trigo

CIP: International Potato Cente - Centro Internacional de la Papa

IBSRAM: International Board for Soil Research and Management

ICARDA: International Center for Agricultural Research in the Dry Areas

ICIPE: International Centre of Insect Physiology and Ecology

ICRA: International Centre for Development Oriented Research in Agriculture

ICRAF: International Centre for Research in Agroforestry

ICRISAT: International Crops Research Institute for the Semi-Arid Tropics

IFPRI: International Food Policy Research Institute

IIBC: International Institute of Biological Control

IITA : International Institute of Tropical Agriculture

ILRI: International Livestock Research Institute

IPGRI: International Plant Genetic Resources Institute

IRRI: International Rice Research Institute

ISAAA: International Service for the Acquisition of Agri-biotech Applications

ISNAR: International Service for National Agricultural Research

IWMI: International Water Management Institute

ZIL: Centre suisse pour l'agriculture internationale 


\section{RÉFÉRENCES}

Banque mondiale, 2001, Rapport sur le développement dans le monde 2000-2001. Combattre la pauvreté.

Becker B., 2001, Oeffentliche Agrarforschung - wohin? Wie werden die Schwerpunkte in der öffentlichen Forschung gesetzt und unter welchem Druck steht die öffentliche Forschung in der Schweiz?, exposé présenté lors de la rencontre «Agrarforschung - für wen?» de Swissaid et de la Déclaration de Berne, Berne, 12 octobre.

CGIAR, 2000, CGIAR Annual Report 2000 : The Challenge of Climat Change: Poor Farmers at Risk.

Dalrymple D., 2001, International Agricultural Research as Global Public Good: A Review of Concepts, Issues, and the CGIAR Experience, Draft n 11 , October 26 (non publié).

DDC, 1994, Lignes directrices Nord-Sud.

—, 1999, Politique sectorielle dans le domaine agricole.

—, 2000, Stratégie 2010 de la DDC.

Delgado Christopher L., Rosegrant Mark W., and Meijer Ssiet, 2001, Livestock to 2020: The Revolution Continues, Ag-Trade, International Agricultural Trade Research Consortium, <www.agtrade.org/ digests/digest33.htm>.

ETHZ, 1996, Leitbild der ETH Zürich, <www.ethz.ch/overview/profile_de.asp>.

FAO, 2000, La situation mondiale de l'alimentation et de l'agriculture.

Fuglie K., Narrod C., and Neumeyer C., 2000, «Public and Private Investment in Animal Research», in Fuglie K. and Schimmelpfenning D. (eds.), Public-Private Collaboration in Agricultural Research: New Institutional Arrangements and Economic Implications, Iowa State University Press.

IFAD, 2000, Rural Poverty Report 2000.

IRRI, 2001, "Golden Rice» Arrives in Asia, IRRI Media Hotline, 26 January, <www.irri.org/vis/ line2001.htm\#GoldenRice>

IUED, 2000, Annuaire Suisse-Tiers Monde 2000, Genève.

Lipton M., 1999, Reviving Global Poverty Reduction: What Role for Genetically Modified Plants ?, 1999 Sir John Crawford Memorial Lecture.

Loi fédérale sur la coopération au développement et l'aide humanitaire internationales, 1976.

Potrykus I., 2001, «Golden Rice and Beyond», Plant Physiology, vol. 125, pp. 1157-1161, <www. checkbiotech.org/root/index.cfm ?fuseaction=search\&search=rice\&doc_id=604\&start=6>.

Rosegrant Mark W., Paisner Michael S., Meijer Siet, and Witcover Julie, 2001, 2020 Global Food Outlook, Trends, Alternatives, and Choices: A 2020 Vision for Food, Agriculture, and the Environment Initiative, Washington, D.C.: International Food Policy Research Institute, <www.ifpri.org/ 2020conference/publications.asp >.

Sachs J., 1999, «Helping the World's Poorest », The Economist, August.

ZIL, 2000, Annual Report 2000, Centre suisse pour l'agriculture internationale, EPF Zurich, <www.zil.ethz.ch/publications/Annual Report2000.pdf>. 